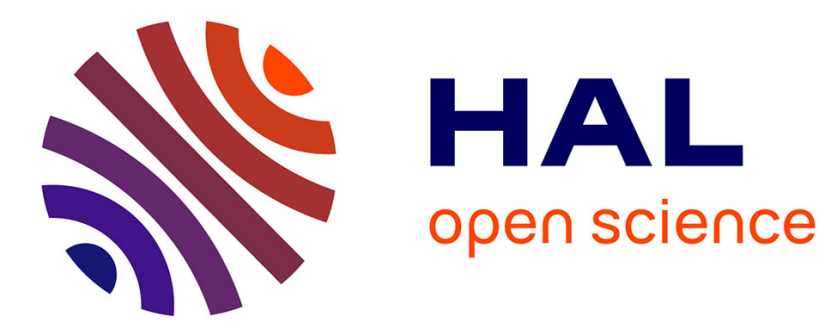

\title{
Interplay between epitaxial strain and low dimensionality effects in a ferrimagnetic oxide
}

Elena Popova, Marwan Deb, Laura Bocher, Alexandre Gloter, Odile Stéphan, Bénédicte Warot-Fonrose, Bruno Bérini, Yves Dumont, Niels Keller

\section{- To cite this version:}

Elena Popova, Marwan Deb, Laura Bocher, Alexandre Gloter, Odile Stéphan, et al.. Interplay between epitaxial strain and low dimensionality effects in a ferrimagnetic oxide. Journal of Applied Physics, 2017, 121 (11), pp.115304. 10.1063/1.4978508 . hal-01707064

\section{HAL Id: hal-01707064 \\ https://hal.science/hal-01707064}

Submitted on 23 Feb 2018

HAL is a multi-disciplinary open access archive for the deposit and dissemination of scientific research documents, whether they are published or not. The documents may come from teaching and research institutions in France or abroad, or from public or private research centers.
L'archive ouverte pluridisciplinaire HAL, est destinée au dépôt et à la diffusion de documents scientifiques de niveau recherche, publiés ou non, émanant des établissements d'enseignement et de recherche français ou étrangers, des laboratoires publics ou privés. 


\section{Interplay between epitaxial strain and low dimensionality effects in a ferrimagnetic oxide}

Elena Popova, Marwan Deb, Laura Bocher, Alexandre Gloter, Odile Stéphan, Bénédicte Warot-Fonrose, Bruno Berini, Yves Dumont, and Niels Keller

Citation: Journal of Applied Physics 121, 115304 (2017); doi: 10.1063/1.4978508

View online: https://doi.org/10.1063/1.4978508

View Table of Contents: http://aip.scitation.org/toc/jap/121/11

Published by the American Institute of Physics

\section{Articles you may be interested in}

Bismuth iron garnet $\mathrm{Bi}_{3} \mathrm{Fe}_{5} \mathrm{O}_{12}$ : A room temperature magnetoelectric material

Applied Physics Letters 110, 142404 (2017); 10.1063/1.4979826

Effects of post-deposition annealing on the structure and magnetization of PLD grown yttrium iron garnet films Journal of Applied Physics 121, 113901 (2017); 10.1063/1.4978310

Epitaxial growth of $\mathrm{Y}_{3} \mathrm{Fe}_{5} \mathrm{O}_{12}$ thin films with perpendicular magnetic anisotropy

Applied Physics Letters 110, 202403 (2017); 10.1063/1.4983783

Ferroelectric or non-ferroelectric: Why so many materials exhibit "ferroelectricity" on the nanoscale Applied Physics Reviews 4, 021302 (2017); 10.1063/1.4979015

Pulsed laser deposition of epitaxial yttrium iron garnet films with low Gilbert damping and bulk-like magnetization APL Materials 2, 106102 (2014); 10.1063/1.4896936

Universal rules for visible-light absorption in hybrid perovskite materials Journal of Applied Physics 121, 115501 (2017); 10.1063/1.4978071

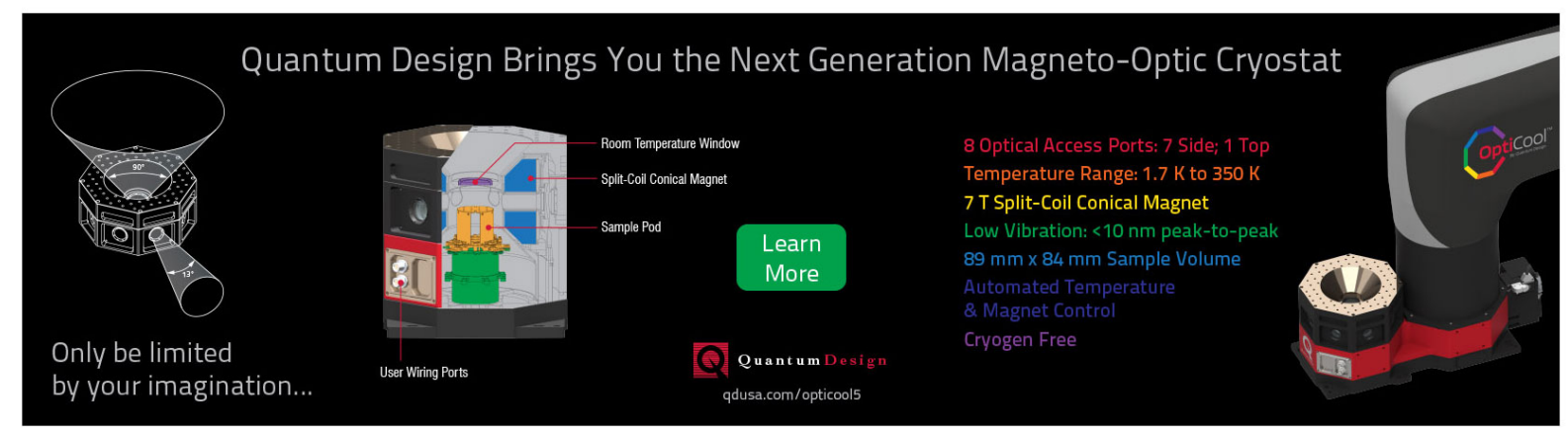




\title{
Interplay between epitaxial strain and low dimensionality effects in a ferrimagnetic oxide
}

\author{
Elena Popova, ${ }^{1, a)}$ Marwan Deb, ${ }^{1}$ Laura Bocher, ${ }^{2}$ Alexandre Gloter, ${ }^{2}$ Odile Stéphan, ${ }^{2}$ \\ Bénédicte Warot-Fonrose, ${ }^{3,4}$ Bruno Berini, ${ }^{1}$ Yves Dumont, ${ }^{1}$ and Niels Keller ${ }^{1}$ \\ ${ }^{1}$ Groupe d'Etude de la Matière Condensée (GEMaC), CNRS-UVSQ, Université Paris-Saclay, 45 avenue des \\ Etats-Unis, 78035 Versailles, France \\ ${ }^{2}$ Laboratoire de Physique des Solides (LPS), CNRS UMR8502, Univ. Paris-Sud, Université Paris-Saclay, \\ 91405 Orsay, France \\ ${ }^{3}$ Centre d'Elaboration de Matériaux et d'Etudes Structurales (CEMES), CNRS, 29 rue J. Marvig, 31055 \\ Toulouse, France \\ ${ }^{4}$ Transpyrenean Associated Laboratory for Electron Microscopy CEMES-INA, CNRS-University of Zaragoza, \\ Toulouse, France and Zaragoza, Spain
}

(Received 4 December 2016; accepted 28 February 2017; published online 20 March 2017)

Thin film properties are strongly influenced by strain and low dimensionality effects, especially when the film thickness is about a few unit cells, which corresponds to the thicknesses targeted in most of contemporary studies. In oxides, these effects are responsible for the dramatic modification of the physical properties that sometimes can change the nature of a material. Nevertheless, it is not always possible to distinguish the contribution of the strain to the changes in physical properties from the contribution due to low dimensional effects. In the present study, bismuth iron garnet $\mathrm{Bi}_{3} \mathrm{Fe}_{5} \mathrm{O}_{12}(\mathrm{BIG})$ is chosen to separate both effects. This material possesses, among other outstanding physical properties, the giant Faraday rotation that allows investigating films with the thickness below a few unit cells. Three series of BIG films of various thicknesses were grown on three isostructural substrates with different lattice parameters: $\mathrm{Y}_{3} \mathrm{Al}_{5} \mathrm{O}_{12}(001), \mathrm{Gd}_{3} \mathrm{Ga}_{5} \mathrm{O}_{12}(001)$, and $(\mathrm{GdCa})_{3}(\mathrm{GaMgZr})_{5} \mathrm{O}_{12}(001)$. The structural, magnetic, and magneto-optic properties were studied as a function of film thickness and strain. Furthermore, critical behavior of the BIG films was investigated in the vicinity of the Curie temperature. The obtained results allowed distinguishing between the low dimensional effects and the strain due to the epitaxial growth. While the size reduction is mostly responsible for the decrease of the magnetic characteristics of the films, the strain influences rather the critical thickness below which the material starts to behave as a low dimensional system. Published by AIP Publishing. [http://dx.doi.org/10.1063/1.4978508]

\section{INTRODUCTION}

Oxide materials are famous for the versatility of their physical properties allowing studying many fundamental effects and leading to a wide range of potential applications. In recent years, the progress in the fabrication of oxide thin films is remarkable. In spite of the lattice mismatch, high quality epitaxial films can be grown on different single crystalline substrates and their properties extensively controlled and fine-tuned. It is now possible to grow ultrathin films (i.e., the films that are a few unit cells thick) of complex oxides that are perfectly stoichiometric and have extremely low roughness. This allows to investigate the thicknessdependent phenomena with great precision, as well as to consider the advanced applications for these materials as single films or combined into heterostructures. ${ }^{1}$

The physical properties of ultrathin films can be very different from those of bulk material or even thick film of the same composition. The main reasons for the property modifications are epitaxial strain and low dimensional effects. Both phenomena become significant as the material thickness decreases and their influence on the film properties

\footnotetext{
a) Author to whom correspondence should be addressed. Electronic mail: olena.popova@uvsq.fr
}

can be comparable for ultrathin films. In the following paragraphs, the influence of each effect is discussed separately.

The control and the comprehension of the strain are extremely important for material properties engineering as it can be used, for example, for the tuning of the physical properties of a material or for the creation of new functionalities in the existing compound ${ }^{2,3}$ such as, for example, transforming a paraelectric antiferromagnet in a ferroelectric ferromagnet. ${ }^{4}$ The role of the strain can be crucial in stabilization of the novel structures and in discovering the novel phenomena, as well as in driving various phase transitions. Strain may lead to changes in microstructure, crystal structure, and film composition. Variable strains can be applied to a thin film using a piezoelectric substrate. This method allows changing gradually the stress applied to a film; unfortunately, it is only suitable for a certain class of oxide materials compatible with the crystal structure of a piezoelectric substrate. The most common way to change the epitaxial strain is to grow a chosen material on the isostructural substrates with different lattice parameter. This approach has been used for the oxides having perovskite structure $^{2,3,5-8}$ and allowed tuning of the multiferroic properties of these materials. As for the garnets, it has been shown ${ }^{9}$ that several iron garnet films present a modification 
of the magnetic anisotropy with the epitaxial strain due to the growth on two types of garnet substrates. Besides, the lattice misfit strain could apparently be responsible for the cerium valence change in $\mathrm{Ce}_{3-\mathrm{x}} \mathrm{Bi}_{\mathrm{x}} \mathrm{Fe}_{5} \mathrm{O}_{12}$ films. ${ }^{10}$

The low dimensional effects are extensively studied in metallic thin films (see, for example, Ref. 11 and references therein) where their influence is detectable in particular, in the modification of the magnetic properties such as saturation magnetization and Curie temperature. The modification of the magnetic characteristics is observed as well in oxide thin films $s^{5}$ and is often inaccurately ascribed to the influence of the epitaxial strain. ${ }^{6,12-14}$ Therefore, it is crucial to be able to separate the strain effects from those originating from the reduction of the material thickness.

In this context, bismuth iron garnet $\mathrm{Bi}_{3} \mathrm{Fe}_{5} \mathrm{O}_{12}(\mathrm{BIG})$ is a model system for the investigation of the finite size effects in a ferrimagnet since it possesses the highest known Faraday rotation in visible spectral range $\left(\sim 17^{\circ} / \mu \mathrm{m}\right.$ at $\left.550 \mathrm{~nm}\right)$ allowing the magneto-optical measurements of even a monolayerthick film in transmission configuration. Moreover, the importance of this material goes beyond the fundamental physics: thin films of magnetic garnets attract much attention, as they are extremely promising for magneto-optic applications, such as sensors for local magnetic fields and currents, magneto-optic modulators, and magnetophotonic crystals. ${ }^{15-18}$ In the material with the potential applications that require low thicknesses, it is very important to know the evolution of the physical properties with the thickness. The synthesis of pure non-substituted BIG needs the deposition conditions far from thermodynamic equilibrium. This material cannot be synthesized in bulk form, but is stabilized on iso-structural substrates by non-equilibrium growth techniques since late eighties (e.g., Refs. 19-21 and references therein). Particular attention should be given to the control of the cation and oxygen stoichiometry of the films as any type of vacancies can modify significantly the magnetic and structural properties of garnets. ${ }^{2-25}$

In order to investigate the influence of the epitaxial strain on BIG properties, three different substrates are chosen: $\mathrm{Y}_{3} \mathrm{Al}_{5} \mathrm{O}_{12}(001)$ (YAG), $\mathrm{Gd}_{3} \mathrm{Ga}_{5} \mathrm{O}_{12}(001)$ (GGG), and $(\mathrm{GdCa})_{3}(\mathrm{GaMgZr})_{5} \mathrm{O}_{12}(001)$ (SGGG). The respective lattice parameters are $1.200 \mathrm{~nm}, 1.237 \mathrm{~nm}$, and $1.248 \mathrm{~nm}$. The mismatch between the expected lattice parameter of BIG $\left(a_{f}=1.262 \mathrm{~nm}\right)$ and those of YAG, GGG, and SGGG is $5.1 \%, 1.9 \%$, and $1.1 \%$, respectively, and initial layers of the film must therefore undergo a compressive stress.

The heteroepitaxial growth and relaxation modes are of great importance for the study of the strain influence on film properties. Especially, the critical thickness above which the film is relaxed should be estimated as it is a function of the strain applied to a film. In the one-dimensional epitaxial interface model ${ }^{26}$ that describes film relaxation (independently from the adopted growth mode), when an atom of the film is deposited on a substrate, it is subjected to two competing forces. The first one is exerted by the neighboring atoms of the film and tends to preserve the natural atom spacings of a film. The second force arises from the substrate atoms that tend to align the incoming film atoms with the substrate atom positions. These competing forces usually result in film growing pseudomorphically (i.e., preserving substrate spacings) on the substrate till several monolayers and then, when the strain exceeds some critical level, relaxing via the formation of the dislocations. The critical film thickness, above which the dislocations are formed, can be roughly estimated using an oversimplified extension of the model to a thickening overlayer ${ }^{27}$

$$
t_{c}=a_{f}\left(\frac{f_{\text {lim }}}{f}\right)^{2}=a_{f}\left(\frac{f_{\text {lim }}}{\left(a_{f}-a_{s}\right) / a_{s}}\right)^{2},
$$

where $a_{f}$ and $a_{s}$ are the film and the substrate lattice parameters, respectively, $f_{\text {lim }}$ is a stability limit for strained pseudomorphic growth, and $f$ is the lattice mismatch between a film and a substrate. $f_{\text {lim }}$ accounts for the ratio of the forces between the film atoms and across the interface film/substrate; it depends on elastic constants and the height of the periodic potential relief of the substrate. ${ }^{27}$ It is evident from Eq. (1) that the critical thickness decreases rapidly with increasing lattice mismatch. Though this approximation works qualitatively for large misfits, generally it cannot be directly compared with experimental data. However, using the value of critical thickness obtained experimentally from rocking curves of $\mathrm{BIG} / \mathrm{GGG}(001),{ }^{28}$ it is possible to estimate the stability limit of identical BIG films on other garnet substrates and then assess the critical thicknesses of BIG on different garnet substrates. Assuming $t_{c}(\mathrm{BIG} / \mathrm{GGG}(001))$ $=20 \mathrm{~nm},{ }^{28}$ the estimated BIG critical thickness on two other substrates is the following: $t_{c}(\mathrm{BIG} / \mathrm{YAG}(001))=3 \mathrm{~nm}$ and $t_{c}(\mathrm{BIG} / \mathrm{SGGG}(001))=67 \mathrm{~nm}$. Therefore, in principle, it should be possible to grow completely strained or completely relaxed BIG films in the thickness range from $5 \mathrm{~nm}$ to $65 \mathrm{~nm}$, which suits the purpose to separate the influence of the strain from that of low dimensional effects in thin films.

Here macroscopic and microscopic properties of a complex ferrimagnetic oxide are investigated as a function of applied epitaxial strain and film thickness. The local probes, such as (scanning) transmission electron microscopy ((S)TEM imaging techniques) and large scale experimental techniques (X-ray diffraction (XRD) and magneto-optics), are combined in order to get a better understanding of the structural and physical properties at complementary length scales.

The manuscript is organized as follows. The details of film growth, methodology, and the performed experiments are described in Section II. Section III contains the results and the discussion separated in three different chapters: the first one describes the film quality and stoichiometry effects, the second addresses the strain relaxation, and the last deals with finite size effects. The conclusions of the present study are given in Section IV.

\section{EXPERIMENT}

Three series of BIG thin films were grown by pulsed laser deposition on three different garnet substrates: YAG(001), GGG(001), and SGGG(001). A complete study of the BIG film properties has been conducted previously $^{28,29}$ in order to determine the growth conditions needed to obtain the oxygen and cation stoichiometric samples. 
The target has been synthesized with a slight excess of bismuth in order to compensate the cation loss during ablation due to bismuth volatility. ${ }^{21}$ An ultra-high vacuum chamber with base pressure of $5 \times 10^{-9}$ Torr was used for film growth. All the films were deposited with a $\mathrm{KrF}$ excimer laser (wavelength, pulse duration, and frequency of $248 \mathrm{~nm}$, $20 \mathrm{~ns}$, and $1 \mathrm{~Hz}$, respectively) at the temperature of $950 \mathrm{~K}$. A target-to-substrate distance was always kept at $5 \mathrm{~cm}$.

In order to study the bismuth off-stoichiometry effects which can be observed in BIG films, a slight variation in cation stoichiometry between the series on $\mathrm{GGG}(001)$ and on two other garnet substrates was induced via different oxygen pressure and laser fluence. The fluence was maintained at about $2.1 \mathrm{~J} / \mathrm{cm}^{2}$ for all depositions on $\mathrm{GGG}(001)$ and at $1.2 \mathrm{~J} / \mathrm{cm}^{2}$ for the growth on YAG(001) and SGGG(001). BIG/GGG(001) (respectively, BIG/YAG(001) and BIG/SGGG(001)) films were grown in oxygen pressure of $4 \times 10^{-2}$ Torr (respectively, $5 \times 10^{-3}$ Torr), regulated by a flow meter. After the deposition, each sample was cooled down in the growth oxygen atmosphere and without any post-deposition annealing. Within each series, the influence of target erosion (that can lead to slight stoichiometry changes in films and modify film properties) has been minimized by growing thin and thick films alternatively.

The only parameter that was varied within a series was the film thickness, which was ranged from a few unit cells of BIG to several hundreds of nanometers. All the samples prepared for the present study possess stoichiometric oxygen content. The series on YAG and SGGG substrates have stoichiometric cation ratio $\mathrm{Bi} / \mathrm{Fe}=0.6$. This ratio for the series of BIG on GGG substrates is inferior to 0.6. The slight difference in cation stoichiometry hardly affected the strain. Contrary to BIG/GGG films, the physical properties of ultrathin films grown on YAG and SGGG substrates were not measurable below $10 \mathrm{~nm}$.

Film thicknesses were estimated in situ by spectroscopic ellipsometry (SENTECH) and measured ex situ by a VEECO Dektak step-meter. The X-ray diffraction measurements were performed using Siemens D5000 and PANalytical X'Pert PRO diffractometers with $\mathrm{Cu}-\mathrm{K} \alpha$ radiation. Further local structural analyses were performed via (scanning) transmission electron microscopy experiments. Cross-sectional electron transparent samples were prepared by focused ion-beam on either a Helios or a SCIOS dual-beam platform (FEI) following a standard procedure. The sample quality was first controlled by high-resolution transmission electron microscopy (HRTEM) (not shown here). Low-magnified high-resolution and atomic resolution high-angular annular dark-field (HAADF) STEM images were acquired on an aberration corrected (Cs) NION UltraSTEM 200 operated at $200 \mathrm{keV}$. Geometric phase analysis (GPA) was carried out from highangle annular dark field HAADF-STEM images to study the strain distribution in the BIG films and the evolution of the local lattice parameters through the film/substrate interfaces. The FRWRtools of DigitalMicrograph plug-in were used to perform the GPA, ${ }^{30}$ with a mask size of ca. $1 / 2 a_{s}$. In STEM images, the slow scan direction induces scanning distortions that affect the GPA results of the corresponding direction. Here, to overcome this issue, we acquired systematically in the same region of interest a set of two HAADF-STEM images rotated by $90^{\circ}$ with respect to each other. ${ }^{31}$ This enables us to extract by GPA both in-plane and out-of-plane components, always parallel to the fast scan direction avoiding processing artifacts.

Room temperature Faraday rotation and ellipticity $\left(\Theta_{F}\right.$, $\varepsilon_{F}$ ) measurements were carried out using a custom-designed magneto-optical magnetometer (for details see Refs. 21, 24, 32 , and 33) in the field range $\left[-1.0 \times 10^{4} \mathrm{Oe} ; 1.0 \times 10^{4} \mathrm{Oe}\right]$. The magnetic field was applied perpendicular to the film plane. Measurements were performed either at fixed photon energies in variable magnetic field or in a spectroscopic mode at $8 \times 10^{3}$ Oe with a second setup based on a phase modulation technique using a commercial CCD spectrometer. Faraday rotation value at $300 \mathrm{~K}$ is obtained from the magneto-optic hysteresis loops measured at $300 \mathrm{~K}$ for each sample. When necessary, the linear contribution of the substrate was subtracted from the experimental data. Curie temperature was determined from Faraday hysteresis loop measurements on the samples mounted into custom-designed furnace operating from room temperature to $800 \mathrm{~K}$. Quantum Design vibrating sample magnetometer (VSM) in physical properties measurement system (PPMS) environment was used to measure hysteresis curves for BIG/YAG(001) samples in the field range $\left[-3.0 \times 10^{4} \mathrm{Oe} ; 3.0 \times 10^{4} \mathrm{Oe}\right]$. The magnetic field was applied parallel to the film plane.

\section{RESULTS AND DISCUSSION}

\section{A. Film quality and stoichiometry}

The structural quality of the BIG films was verified using XRD measurements. All the films of the three series were single phase epitaxial bismuth iron garnet with cubeon-cube epitaxial relationship: (001)[100 $]_{\mathrm{BIG}} / /(001)[100]_{\text {subs }}$ within the XRD detection limit (inset to Fig. 1). No other phase containing bismuth or iron or their combination was detected within the precision of XRD setup (Fig. 1).

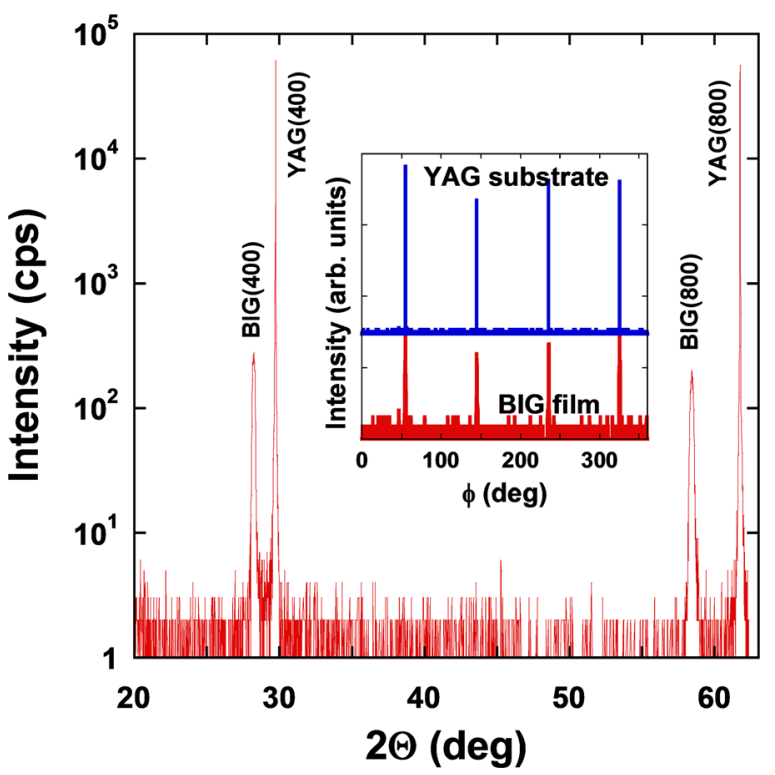

FIG. 1. Typical XRD $\Theta-2 \Theta$ measurement for a BIG/YAG(001) film. Inset shows a $\varphi$-scan for (444) planes of the same film. 
The preservation of BIG properties was verified for the ultrathin films. Figure 2 represents several Faraday ellipticity spectra measured for the $\mathrm{BIG} / \mathrm{GGG}(001)$ films of different thickness. It is obvious that even two unit cell thick film is pure BIG: the characteristic bismuth iron garnet peaks are present for all films (see also Ref. 32 for further details).

The influence of oxygen stoichiometry on BIG properties has been extensively studied previously in our samples. ${ }^{29}$ The observed spectral signature is representative of the crystal field environment of iron ions in both octahedral and tetrahedral oxygen coordination. ${ }^{34,35}$ Therefore, the oxygen environment is stoichiometric. Furthermore, the strong spin-orbit coupling, related to the presence of bismuth ions, is evidenced via the increased Faraday rotation, as described in Refs. 32, 34, and 36.

Bismuth stoichiometry can be further quantified from Curie temperature $T_{C}$ of the films. Indeed, it has been proven ${ }^{21}$ that $T_{C}$ is extremely sensitive to bismuth content in a film. Curie temperature was determined as a function of film thickness for three series of BIG films on different garnet substrates (Fig. 3). For this purpose, magneto-optic hysteresis loops were measured at different temperatures in the range [300 K; $800 \mathrm{~K}$ ]. This type of measurements tends to slightly overestimate the Curie temperature due to the applied magnetic field. The different film stoichiometry is clearly visible in the difference of $T_{C}$ for $\mathrm{BIG} / \mathrm{GGG}(001)$ films from two other series. $T_{C}$ of bulk BIG/GGG(001) films is about $30 \mathrm{~K}$ higher than the Curie temperature of $\mathrm{BIG} / \mathrm{YAG}(001)$ and BIG/SGGG(001) which are very close to estimated stoichiometric value. Horizontal solid (dashed-dotted) line represents Curie temperature for stoichiometric (off-stoichiometric) BIG film from Ref. 21 and references therein.

The deviation from nominal $\mathrm{Bi} / \mathrm{Fe}$ ratio for $\mathrm{BIG} / \mathrm{GGG}$ films should also be visible in the values of the lattice parameters. The out-of-plane lattice parameter was calculated from $\Theta-2 \Theta$ diffraction scans as a function of film

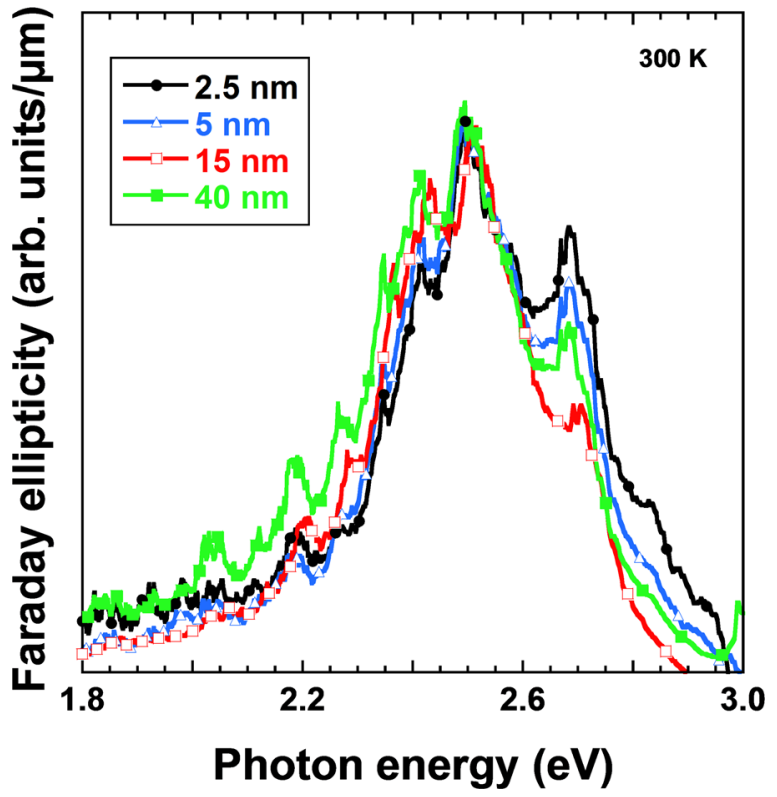

FIG. 2. Room temperature Faraday ellipticity spectra for the series of BIG/ GGG(001) films of different thickness.

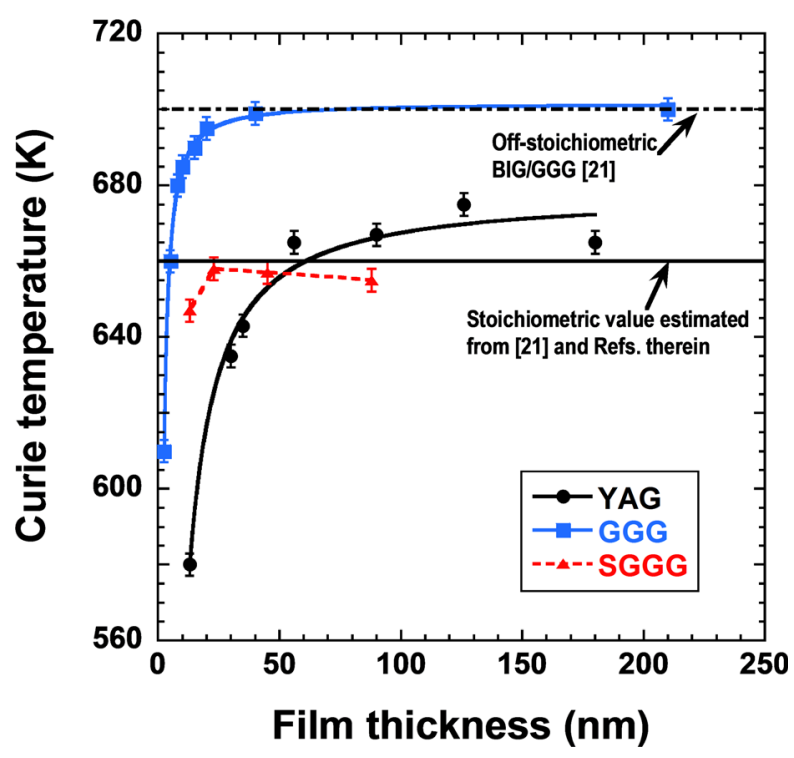

FIG. 3. Curie temperature as a function of film thickness for three series of BIG films on different garnet substrates. For the BIG/YAG(001) and BIG/ GGG(001) films, the solid lines are the adjustments with the equation $T_{C}(t)=T_{C}^{\text {bulk }}\left\{1-\left(\frac{\xi_{0}}{t}\right)^{\lambda}\right\}$. For BIG/SGGG(001), the line is a guide for the eye. Horizontal solid (dashed-dotted) line represents Curie temperature for stoichiometric (off-stoichiometric) BIG film.

thickness for the three series of BIG films on different substrates (Fig. 4). The expected "bulk" value of the lattice parameter is represented by continuous horizontal line in Fig. 4. This stoichiometric value was obtained extrapolating data from bismuth-substituted yttrium iron garnet to complete Bi substitution. ${ }^{21,37,38}$ The lattice parameter of thicker BIG films on YAG and SGGG substrates are close to the expected value. The films on GGG substrates are slightly

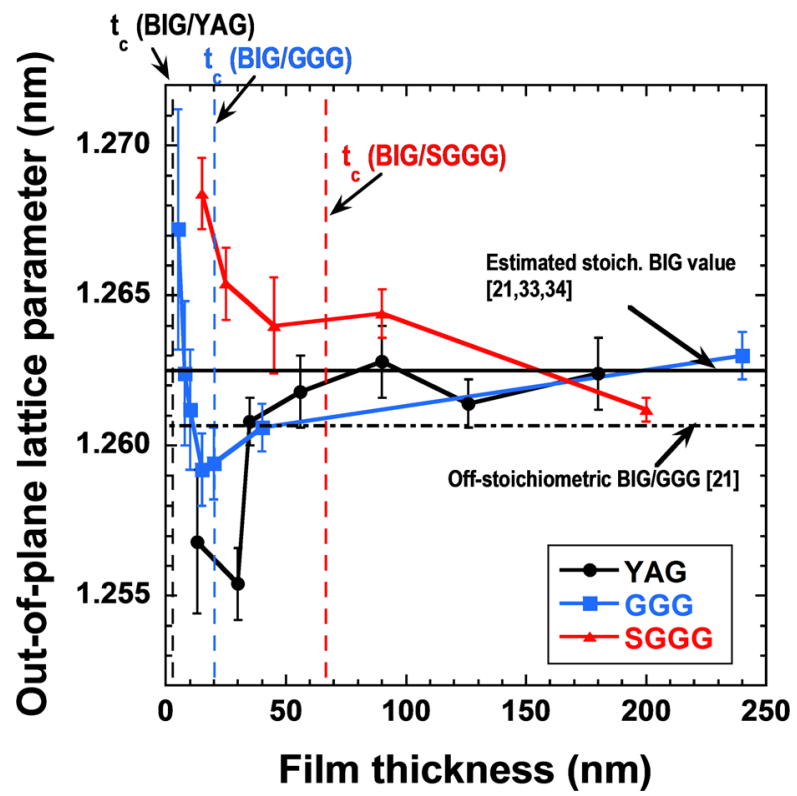

FIG. 4. Out-of-plane lattice parameter determined from XRD measurements for three series of BIG films deposited on YAG(001), GGG(001), and SGGG(001) substrates. Horizontal solid (or dashed-dotted) line designates the value of stoichiometric (or off-stoichiometric) BIG lattice parameter. Vertical dashed lines correspond to the critical thickness estimated from Eq. (1) for the film grown on different substrates (see text for details). 
off-stoichiometric and on the overall have smaller lattice parameter than the expected one and therefore should be compared with the value represented by the dashed-dotted black line in Fig. 4, which corresponds to the bismuth content determined from Curie temperature measurements (Fig. 3) according to Ref. 21.

To summarize, all the three series consist of oxygenstoichiometric single crystalline BIG. The series on GGG substrate are slightly off-stoichiometric in bismuth, i.e., Bi/ $\mathrm{Fe}<0.6$.

\section{B. The role of the strain}

\section{Strain relaxation}

According to the lattice mismatch between the films and the substrates, all the films are presumed to undergo a compressive epitaxial strain. Due to the compressive in-plane strain, it is thus expected for the strained part of a film that the out-of-plane lattice parameter should be larger than the bulk value represented by continuous horizontal line in Fig. 4. This is indeed the case for the films grown on GGG and SGGG substrates. The ultrathin films on YAG substrates show the inverse tendency: their out-of-plane lattice size is smaller than bulk value for the thicknesses below $60 \mathrm{~nm}$. The estimated critical thickness, shown in Fig. 4 for three series by vertical dashed lines, seems to be in agreement with XRD data for BIG/SGGG and BIG/GGG, though it is rather difficult to link with the data obtained for the films grown on YAG. A mechanism of lattice relaxation different from the one described in introduction must take place for BIG/YAG films. Furthermore, all the films having thicknesses above $60 \mathrm{~nm}$ are relaxed and have lattice parameter close to the value estimated for BIG (Fig. 4).

The relaxation mechanisms of ca. $80 \mathrm{~nm}$ thick BIG films grown on different substrates were studied in detail using HRTEM (not shown here) and Cs-STEM. In the frame of the simplified model of accommodation of the lattice mismatch-apart from strained film growth-a dislocation is expected every $x_{0}=a_{f}\left(a_{f}-a_{s}\right)$ planes. ${ }^{39,40}$ This means that the expected numbers of planes separating two dislocations are: between 20 and 21 for BIG/YAG, between 52 and 53 for $\mathrm{BIG} / \mathrm{GGG}$, and around 90 for BIG/SGGG. These distances correspond to $13 \mathrm{~nm}$ (10 unit cells), $33 \mathrm{~nm}$ (25 unit cells), and $57 \mathrm{~nm}$ (44 unit cells) between eventual dislocations on different substrates. The lattice deformation, corresponding to the strain, was determined using GPA on CsSTEM images. This method allows the determination of local displacements and strain fields in a specimen from experimental images. ${ }^{41}$

Figure 5 summarizes GPA calculated from Fig. 5(a) for BIG/SGGG films. The evolution of the in-plane lattice parameter from the film/substrate interface toward the top of the film evidences a smooth continuous strain relaxation over a distance of typically $20 \mathrm{~nm}$ (Figs. 5(b) and 5(d)). A lattice parameter of $1.270 \mathrm{~nm}$, which is ca. $1.8 \%$ larger than the inplane lattice parameter of the SGGG substrate $(1.248 \mathrm{~nm})$, is reached for the thickness above $20 \mathrm{~nm}$. Such value is in good agreement with the $1.1 \%$ difference expected for a BIG relaxed structure as compared with the SGGG substrate. The change of out-of-plane strain is more abrupt at the film/substrate interface, as presented in Figs. 5(c) and 5(d). Nevertheless, the GPA evidences a rapid relaxation of the out-of-plane lattice distance in the thin film in agreement with the XRD data. In the vicinity of the interface the out-ofplane lattice is locally strained till $2.3 \%$ and then it converges to the expected relaxed lattice of BIG. This confirms a rather strong compressive strain of the BIG film grown on SGGG occurring at the interface. These GPA maps also evidence (Fig. 5(b)) the presence of dislocations occurring from 10 to $20 \mathrm{~nm}$ from the interface in concordance with the strain relaxation. Furthermore, an atomically resolved STEM-HAADF image of BIG/SGGG interface can be seen in Fig. 6(a) and confirms a cube-on-cube interface, with an absence of dislocations at the interfacial plane.

The XRD measurements of the BIG/YAG film suggest a different relaxation mechanism. According to the STEM investigation, the relaxation is realized through two different mechanisms within different crystallographic grains: Vernier of misfit (Fig. 6(b)) and the tilted growth (Fig. 6(c)). In the following, these two relaxation mechanisms are discussed in detail.

Figure 6(b) shows a typical HAADF-STEM image of $\mathrm{BIG} / \mathrm{YAG}$ interface with the first type of lattice relaxation. The lattice mismatch between BIG and the garnet substrate
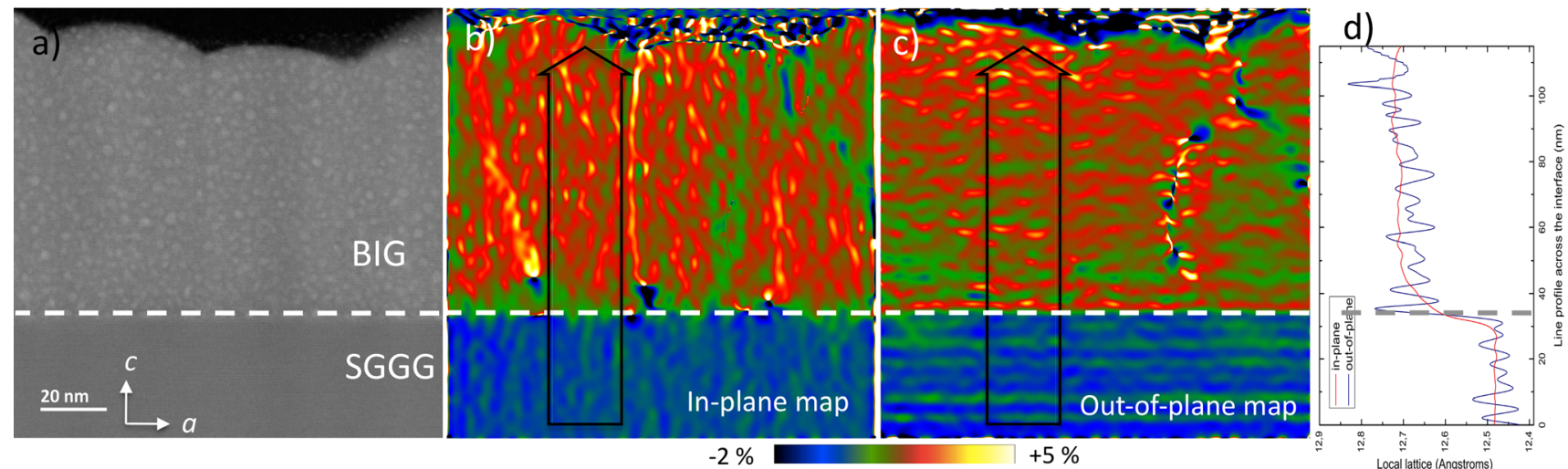

FIG. 5. Local strain analysis in a BIG/SGGG(001) film. (a) Low-magnified high-resolution HAADF-STEM image of the interface. GPA maps of the (b) in-plane and (c) out-of-plane lattice parameters determined from image (a) relative to SGGG substrate with $a=1.248 \mathrm{~nm}$. The black arrows in (b) and (c) correspond to the profile size reported in (d). 


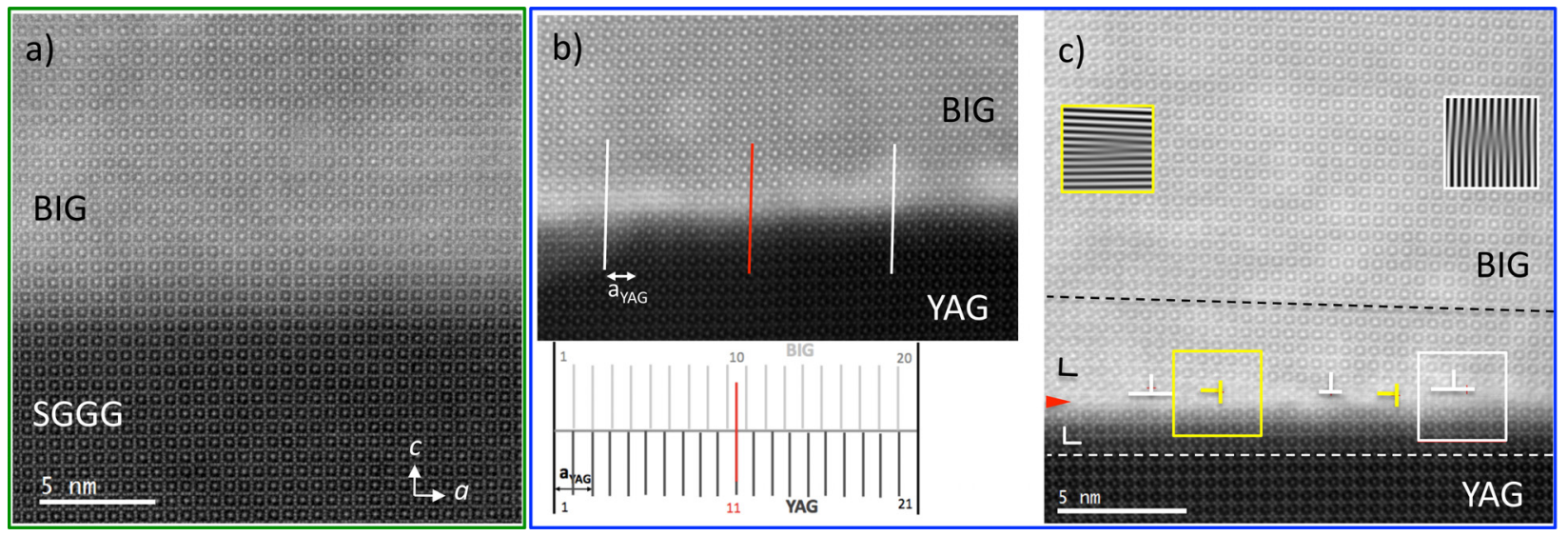

FIG. 6. Atomically-resolved HAADF-STEM images of the interfaces BIG/substrate. (a) BIG/SGGG(001) interface. (b) BIG/YAG(001) interface within a nontilted grain. The lines illustrate the relaxation via Vernier of misfit which is schematically presented in the inset. (c) BIG/YAG(001) interface within a tilted grain. Burgers vectors localize the in-plane (yellow) and out-of-plane (white) dislocations at the interface. The inset figures present examples of the inverse Fourier Transforms to highlight the presence of the in-plane and out-of-plane dislocations.

is adapted via so-called Vernier of misfit. ${ }^{27}$ Indeed, BIG and YAG columns match almost perfectly every 20 (21) half unit cells of BIG (YAG), which correspond to approximately 10 unit cells. The schematic representation of this relaxation process is presented in the inset to Fig. 6(b) for better clarity. No periodic dislocation is observed (as could be expected from the domain matching epitaxy concept ${ }^{40}$ ). This type of relaxation occurs when interfacial bonding between a film and a substrate is much weaker than the bond strength within the film or the substrate. ${ }^{27}$ In this case, the crystals tend to preserve their natural lattice spacings, with different lattice periodicities resulting in a Vernier of misfit, i.e., coherent interface with some very weak residual elastic strain as shown by changes in lattice parameters for ultrathin films (Fig. 4). For such 20 (21) periodicity, a residual strain of ca. $0.1 \%$ is expected. This preferential bonding between the atoms of the film gives rise to the three-dimensional growth mode of BIG films on garnet substrates observed previously. ${ }^{42}$ The GPA results for the Vernier relaxation mechanism are resented in Fig. 7. According to out-of-plane lattice and the lattice rotation maps shown in Figs. 7(b) and 7(c) and the profiles of the local in-plane and out-of-plane lattices, the relaxation of the BIG films occurs immediately at the interface suggesting fast strain relaxation.

As shown previously (Fig. 4), there is a difference in the out-of-plane lattice parameter of ultrathin BIG grown on GGG or SGGG and on YAG: the lattice decreases with increasing film thickness for BIG/GGG and BIG/SGGG and increases for BIG/YAG, suggesting a possible small tensile strain for the latter. This is arising from the second BIG/ YAG relaxation mechanism, as reported in Figs. 6(c) and 8. Two grain boundaries-marked with white arrows-are clearly visible in Fig. 8(a) and the GPA maps in Figs. 8(b) and $8(\mathrm{c})$. Figure $8(\mathrm{c})$ represents the GPA lattice rotation map calculated from the STEM-HAADF image of Fig. 8(a), using the substrate as a reference. From this analysis it is clear that the middle grain, i.e., grain 2 , is tilted by approximately $2.2^{\circ}$ with respect to the surrounding grains (1 and 3) and the substrate. The grain tilt is also confirmed from the fast Fourier transform (FFT) of the film and the substrate (Fig. 8(d)): non-tilted grains and the substrate have the same orientation and the lattice of the tilted grain is slightly rotated. Such rotation can accommodate only a fraction of the lattice mismatch from the YAG substrate. Nevertheless, the in-plane and out-of-plane strains (Fig. 8(e)) are relatively uniform and relax rapidly. The rest of the strain is then also accommodated by a series of dislocations at the interface. The periodicity of this dislocation network corresponds to every 17 (18) half BIG (YAG) unit cells. Figure 6(c) evidences a strong reconstruction at the interface between a BIG tilted grain and the YAG substrate highlighting an example of the presence of these dislocations. It has to be noted that such mechanism of relaxation via 17 (18) dislocations series and $2.2^{\circ}$ tilt induces a small residual tensile strain. This is confirmed by the in-plane and out-of-plane lattice profile in Fig. 8(e) that shows a slightly reduced out-of-plane lattice over the first $30 \mathrm{~nm}$ (c/a ratios of typically 0.99 are obtained in this area).

This estimation of the lattice relaxation (Fig. 8(e)) is consistent with the XRD data (Fig. 4), where the out-of-plane lattice parameter of the films was inferior to the "bulk" value for the thicknesses below $50 \mathrm{~nm}$. For the strong lattice mismatch between BIG and YAG, the relation $18 a_{s}>17 a_{f}$ holds leading to the slight in-plane tensile stress and lattice decrease for low film thicknesses observed in XRD measurements.

To conclude, for BIG/SGGG films and most probably for BIG/GGG films having a small lattice mismatch, the relaxation mechanism is a compressive strain over several nanometers with a relaxation mechanism based on dislocation appearance within a grain. In the case of BIG/YAG films, two relaxation mechanisms coexist. The first one involves an epitaxial domain matching (or Vernier misfit) with 20 (21) periodicity and the second is related to the presence of tilted grain with dislocation 17 (18) network and a residual small tensile strain. It should be noted that whatever the substrate, the BIG film is relaxed for the thicknesses above about sixty nanometers. Therefore, for the studied film thicknesses, there is a range in film thickness where the films are definitely relaxed, though this range does not correspond to the expected value. 

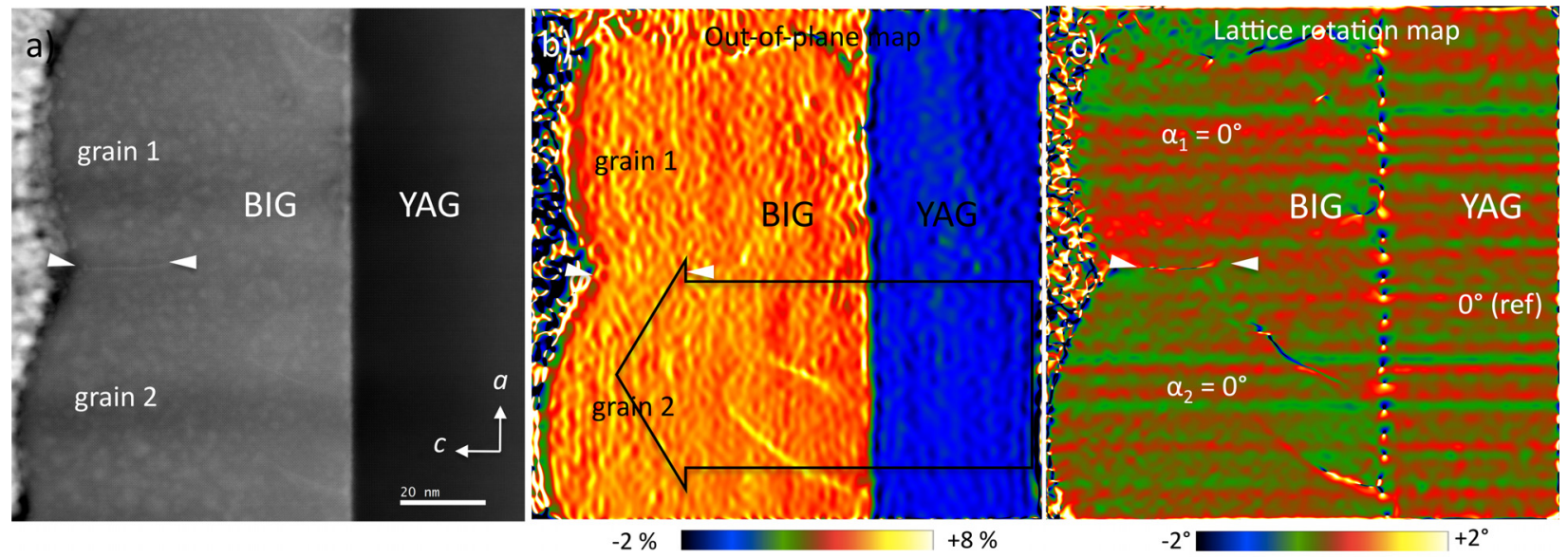

d)

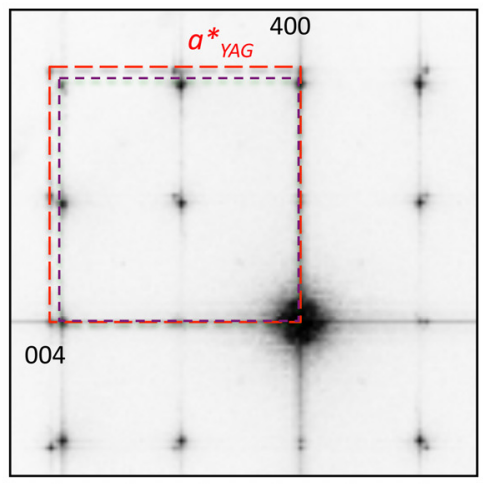

e)

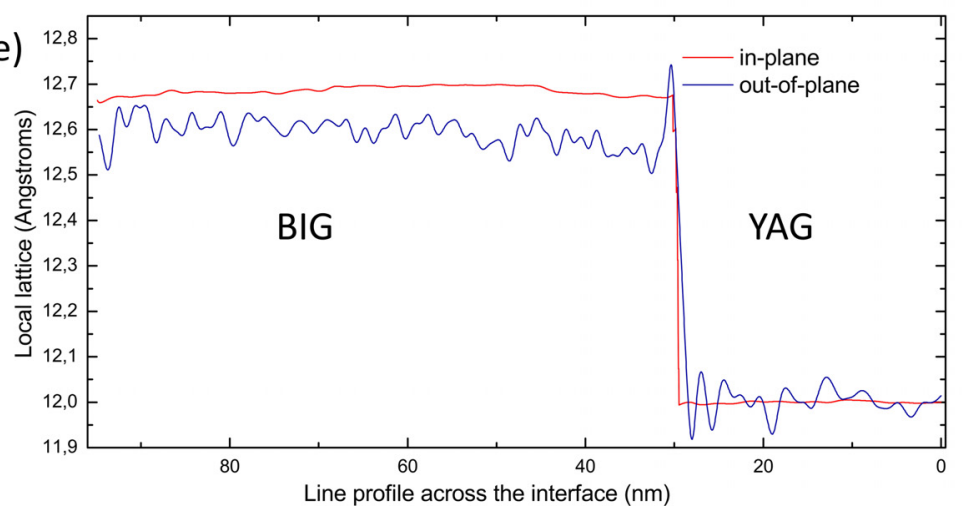

FIG. 7. Non-tilted grains in a BIG/YAG(001) film. (a) Low magnified high resolution HAADF-STEM image of the interface. White arrows mark grain boundaries. GPA maps of the (b) out-of-plane lattice parameter and (c) the lattice rotation determined from image (a) with respect to the substrate. The black arrow in (b) corresponds to the profile size in (e). (d) FFT of the image (a) including BIG film and YAG substrate, showing the splitting of the diffraction spots. BIG and YAG diffraction patterns are marked by violet and red dashed squares, respectively. (e) Local in-plane and out-of-plane lattice parameters within a non-tilted grain.

\section{Influence of the strain on physical properties}

The influence of the strain is determined comparing the physical characteristics of the ultrathin BIG films on different substrates at a given film thickness.

As seen in Fig. 3, for all series, the Curie temperature decreases with decreasing film thickness. However, this decrease takes place more rapidly in case of large lattice mismatch between the film and the substrate: $T_{C}$ starts to decrease below $50 \mathrm{~nm}$ for BIG/YAG, below $30 \mathrm{~nm}$ for BIG/ GGG, and below $20 \mathrm{~nm}$ for BIG/SGGG.

Faraday rotation of all films was measured at room temperature using the wavelength of $550 \mathrm{~nm}$. Faraday hysteresis curves were recorded with the field applied perpendicularly to the film plane. Figure 9(a) summarizes the measurement results for three series of BIG films as a function of film thickness. For each series, the saturation Faraday rotation value decreases with decreasing film thickness. For the ultrathin films, $\Theta_{F}(\mathrm{BIG} / \mathrm{SGGG})>\Theta_{F}(\mathrm{BIG} / \mathrm{GGG})>\Theta_{F}(\mathrm{BIG} / \mathrm{YAG})$, as for corresponding lattice parameters (Fig. 4). The properties of films grown on YAG and SGGG substrates were unmeasurable below $10 \mathrm{~nm}$, most probably due to film morphology (higher roughness due to island growth). The increase of Faraday rotation was observed for BIG films with $t<10 \mathrm{~nm}$ grown on GGG substrates. This effect can be due to the changes in electronic properties at the lowest thicknesses.
The saturation magnetization of the thin films grown on GGG and SGGG substrates and having thickness below $350 \mathrm{~nm}$ cannot be measured with conventional magnetometry setups because of the huge paramagnetic contribution from gadolinium ions. The magnetization value at room temperature can be estimated from magneto-optical measurements assuming that a film saturates at the applied magnetic field corresponding to $4 \pi M_{S}+H_{K}$ (where $H_{K}$ is magnetocrystalline anisotropy). Taking into account that in garnets $H_{K} \ll 4 \pi M_{S}$, the contribution of the magnetocrystalline anisotropy can be neglected. ${ }^{28}$ Then, the field at which a film becomes saturated corresponds roughly to the saturation magnetization value. Figure 9(b) shows the saturation magnetization as a function of film thickness for three series of BIG films. The magnetic field was applied perpendicular to the film plane. To check the validity of the used approximation, the saturation magnetization as a function of film thickness was measured at $300 \mathrm{~K}$ for $\mathrm{BIG} / \mathrm{YAG}(001)$ samples using PPMS. The magnetic field was applied parallel to the film plane. The results are in good agreement with the estimated $4 \pi M_{S}$, except for the thinnest BIG film, where the approximation seems not to be valid anymore indicating the presence of an additional anisotropy, most probably due to the film morphology and the relaxation mode. Indeed, though the film is continuous, it presents 3D grains (TEM image not shown here) on the surface that can be the reason 

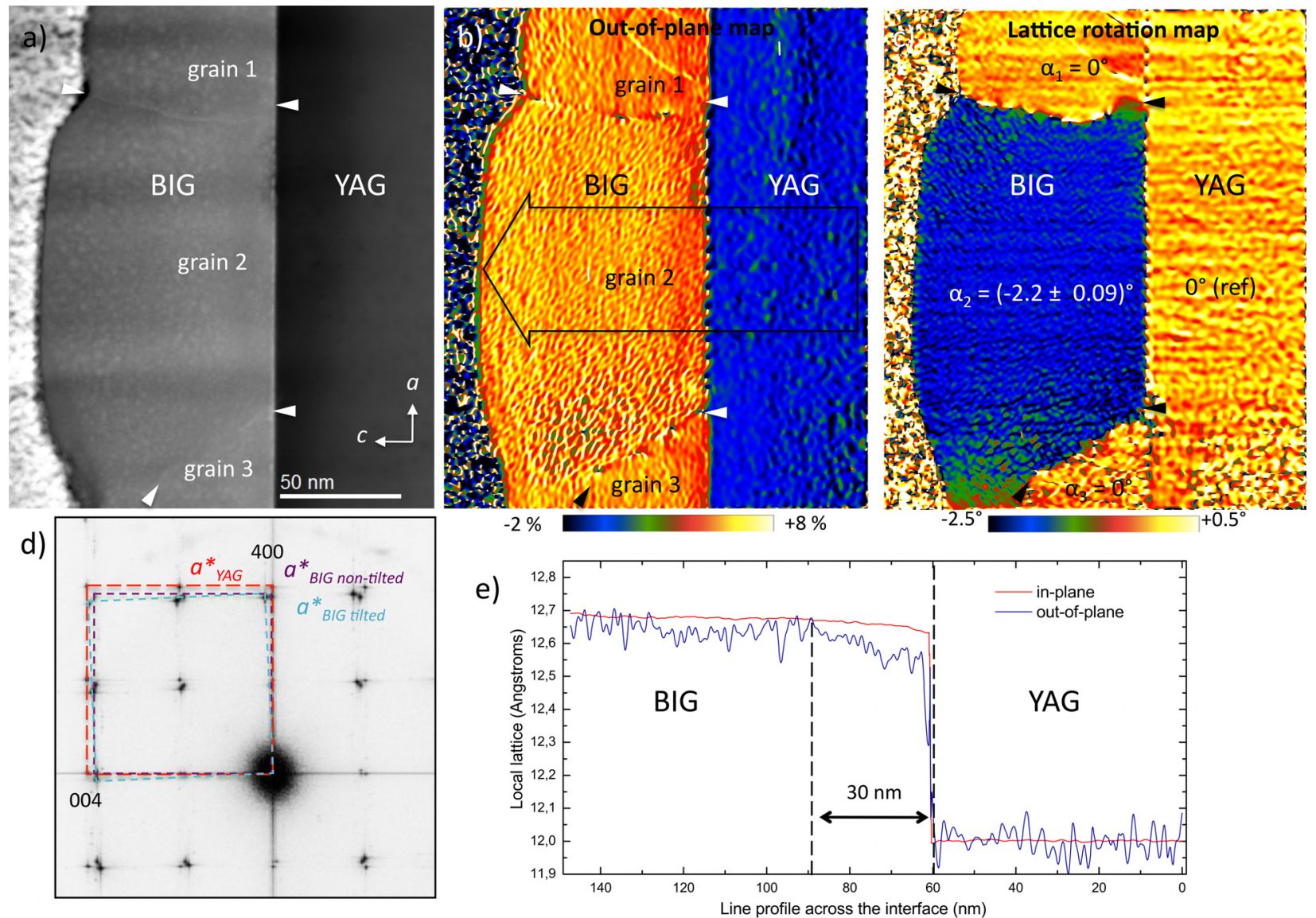

FIG. 8. Tilted grain between non-tilted grains in a BIG/YAG(001) film. (a) Low magnified high resolution HAADF-STEM image of the interface. The white arrows mark grain boundaries. GPA maps of the (b) out-of-plane lattice parameter and (c) the lattice rotation determined from image (a) with respect to the substrate. The black arrow in (b) corresponds to the profile size in (e). (d) FFT of the image (a) including different BIG grains and YAG substrate, showing the splitting of the diffraction spots. Non-tilted BIG grains and YAG diffraction patterns are marked by violet and red dashed squares, respectively. Light blue dashed square corresponds to the diffraction pattern of the tilted grain. (e) Local in-plane and out-of-plane lattice parameters within a tilted grain.

of invalidity of the anisotropy approximation used above. Moreover, it has been observed that in ultrathin garnet films the easy magnetization axis can be out-of-plane due to the anisotropies interplay. ${ }^{12,22,28}$ Overall, the saturation magnetization for three BIG series show the same behavior as the Faraday rotation: the decrease with decreasing film thickness and $4 \pi M_{S}(\mathrm{BIG} / \mathrm{SGGG})>4 \pi M_{S}(\mathrm{BIG} / \mathrm{GGG})>4 \pi M_{S}(\mathrm{BIG} / \mathrm{YAG})$ for $t<50 \mathrm{~nm}$

The magnetic and magneto-optic properties depend clearly on strain. The strain relaxation influences the absolute values of lattice parameters and, therefore, the magnetization,
Curie temperature, and Faraday rotation values for the same thickness of BIG on different substrates. There is a range in thickness where the films are relaxed, but Faraday rotation and magnetization are varying, which can be ascribed to the low dimensionality effects.

\section{Finite size effects}

The magnetic and magneto-optic properties depend as well on film thickness. The low dimensional effects are visible through the decrease of physical characteristics of the
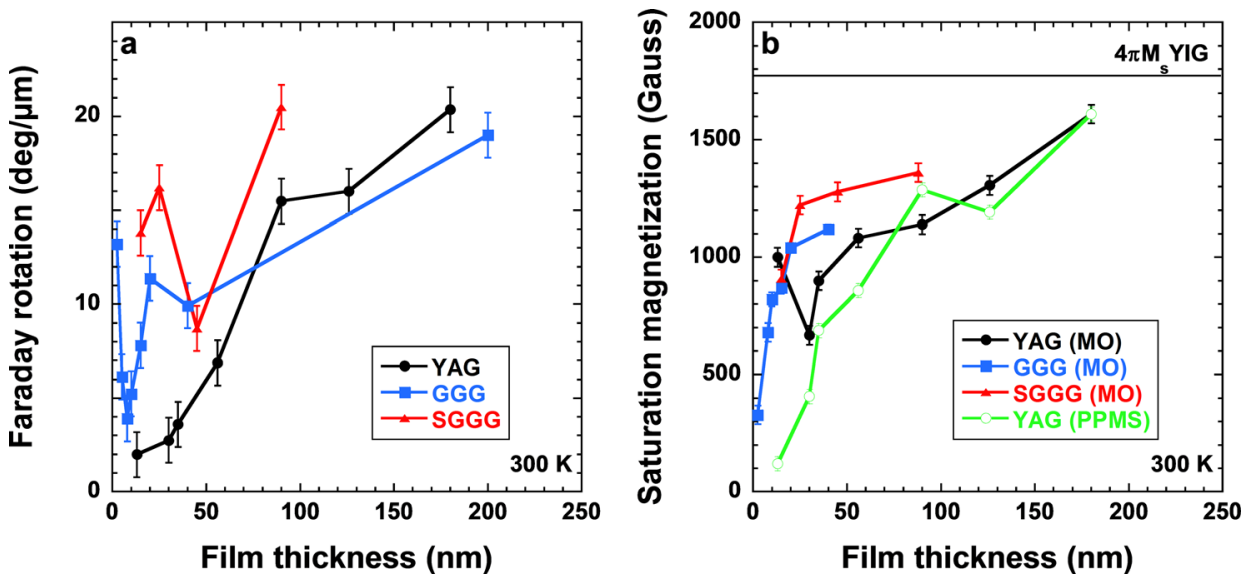

FIG. 9. (a) Faraday rotation of BIG samples grown on different substrates. The measurements were carried out at $300 \mathrm{~K}$ and the wavelength of $550 \mathrm{~nm}$. (b) Saturation magnetization at $300 \mathrm{~K}$ of BIG samples grown on different substrates. The magnetic field was applied perpendicular to the film plane. The saturation magnetization as a function of film thickness was measured for BIG/YAG(001) samples using VSM (open circles) at $300 \mathrm{~K}$. The magnetic field was parallel to the film plane. Horizontal solid line corresponds to the saturation magnetization of yttrium iron garnet. 
films as the function of decreasing thickness within a series. This effect is evident from Figs. 3 and 9, where the magnetization, Faraday rotation, and Curie temperature decrease with decreasing thickness. The slight cation off-stoichiometry of the BIG/GGG series with respect to the two other series does not change qualitatively the dependences of the physical properties on the sample thickness.

Finite-size scaling theory predicts that the critical temperature decreases significantly when one or more dimensions are reduced below a certain characteristic length scale. The scaling law is given by ${ }^{43}$

$$
\frac{T_{C}^{\text {bulk }}-T_{C}(t)}{T_{C}^{\text {bulk }}}=\left(\frac{\xi_{0}}{t}\right)^{\lambda},
$$

where $T_{C}^{b u l k}$ is Curie temperature of a bulk material or a thick film, $T_{C}(t)$ is the Curie temperature of a film of thickness $t$, $\xi_{0}$ is the correlation length in bulk material at $T=0 \mathrm{~K}$, and $\lambda$ is the shift exponent due to the variation of $T_{C}$ for thin films with respect to bulk material. $\lambda$ is inversely proportional to correlation length exponent $\nu$. For magnetic films below a certain critical thickness, the deviation from Eq. (2) was observed and explained by crossover from 3-dimentional to 2-dimentional behavior. ${ }^{44,45}$

Curie temperature variation with film thickness was adjusted for BIG/YAG(001) and BIG/GGG(001) films using Eq. (2). The adjustments are represented in Fig. 3 with solid lines. For BIG/SGGG(001), the line is a guide for the eye, as the data were insufficient for unambiguous adjustment. The resulting critical exponents and correlation lengths are presented in Table I.

To determine the critical exponent for magnetization, the normalized Faraday rotation dependence on the temperature is presented in Fig. 10 for three thick films. Solid lines represent the adjustment of the data with the equation

$$
M(T)=M_{0}\left(T_{C}-T\right)^{\beta},
$$

in the range $\left[0.85 \times T_{C} ; T_{C}\right]$, as critical power function holds for the temperatures: $\left(T_{C}-T\right) / T_{C} \leq 0.15$. The results are shown in Table I for the three films.

The critical exponents describe the behavior of the physical quantities near continuous phase transitions. They depend on the dimension of the system and the range of interaction as well as on spin dimension. As can be seen from Table I, the critical exponents for BIG films grown on YAG, GGG, and SGGG substrates are similar and the values of $\beta$ are close to those determined for YIG thin films $(\beta=0.5)$ that are considered as isotropic 3D magnets with half-integer spin. $^{46}$ However, the correlation length for

TABLE I. Critical exponents and correlation lengths for BIG films on three different garnet substrates determined from data presented in Fig. 9.

\begin{tabular}{lccc}
\hline \hline & BIG/YAG & BIG/GGG & BIG/SGGG \\
\hline$\lambda$ & $1.12 \pm 0.10$ & $1.20 \pm 0.05$ & $\ldots$ \\
$\xi_{0}(\mathrm{~nm})$ & $2.48 \pm 0.18$ & $0.48 \pm 0.02$ & $\ldots$ \\
$\beta$ & $0.51 \pm 0.02$ & $0.47 \pm 0.07$ & $0.53 \pm 0.02$ \\
\hline \hline
\end{tabular}

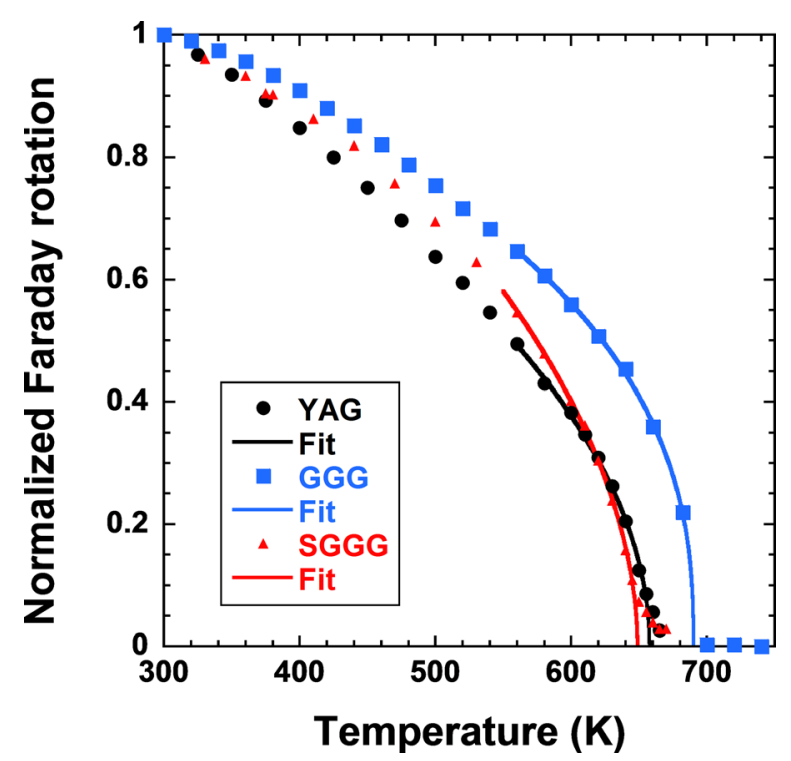

FIG. 10. Faraday rotation, normalized to the value at $300 \mathrm{~K}$, for three thick films of BIG on different substrates. Solid lines represent the adjustment of the data with the equation $M(T)=M_{0}\left(T_{C}-T\right)^{\beta}$ in the range $\left[0.85 \times T_{C} ; T_{C}\right]$.

$\mathrm{BIG} / \mathrm{YAG}$ films is much bigger than for BIG/GGG and, presumably, for BIG/SGGG films. The correlation length of the material is associated to the length scale below which the overall properties of the material begin to differ from those of bulk. ${ }^{47}$ Usually the correlation length is of the order of a few interatomic spacings. However, in experiment, the change in physical properties is observed at higher thicknesses. The actual value of correlation length depends on the external conditions determining the state of the system, such as the temperature and pressure. ${ }^{47}$ The higher correlation length determined for BIG/YAG films is the indication that the film properties start to differ from bulk at higher length scale than for the films grown on two other substrates. This is consistent with the measured physical properties of the three series of BIG films (Figs. 3, 9, and 10). $\xi_{0}^{\lambda}$ gives an indication about the average distance over which the fluctuations of the magnetization are correlated. It may also be interpreted as the number of monolayers under which the Curie temperature vanishes, ${ }^{48}$ which is probably the case in the present study, and corresponds to two unit cells for BIG/YAG and to one-third of the unit cell for BIG/ GGG. The reason for this observation is the different mechanism of the lattice relaxation described above. Moreover, there is no deviation of the experimentally determined Curie temperature for the thinnest films from the fit with Eq. (2) (Fig. 3). Such a deviation is often interpreted as a crossover from two to three-dimensional behavior ${ }^{44,45,49}$ in ultrathin films. Therefore, all the data are consistent with the 3D interactions for the investigated films in the studied thickness range. It should be noted that the film morphology could influence the experimentally determined physical properties. In the present case, the morphologies of BIG/ YAG and BIG/SGGG films are similar and slightly different from BIG/GGG films; however, the results depend rather on the strain. 


\section{The difference between strain and low dimensional effects}

The physical properties variation with the film thickness and lattice strain presented above allows to separate the strain effects from the low dimensionality effects and to take into account the influence of Bi stoichiometry. As expected ${ }^{21}$ the slight deviation from bismuth stoichiometry manifests itself in the reduced value of the lattice parameter and increased Curie temperature for the series on GGG substrates. The influence of the strain is stronger and results in gradual variation of lattice parameter, Faraday rotation, and magnetization as a function of the misfit for the ultrathin films $(\mathrm{t}<50 \mathrm{~nm})$. The low dimensionality effects consist in decrease of $\Theta_{F}, M_{S}$, and $T_{C}$ with decreasing film thickness as observed for many metallic and oxide thin films $\mathrm{s}^{2,5,11}$ and sometimes interpreted as the influence of the strain.

The reasons of the decrease of both magnetization and Curie temperature for ultrathin films of an oxide are still under discussion. ${ }^{2,5}$ It can be argued that the change in the physical properties at low thickness can be explained by the change in the lattice size. The lattice parameter variation presumes, among other effects, a deformation or a tilt of oxygen polyhedra ${ }^{8}$ that can result in the change of the interatomic distances and angles $\mathrm{Fe}-\mathrm{O}-\mathrm{Fe}$. This angle determines the strength of the superexchange interaction ${ }^{25}$ and, therefore, has a great influence on the magnetic properties of an oxide film. However, the magnetic properties of the three series of the films deteriorate independently of the lattice parameter which changes differently for the films grown on YAG and on GGG/SGGG. The most plausible reason for the decrease of the magnetization and Curie temperature must be the same as for the ultrathin metallic films ${ }^{11}$ and related to the break in symmetry at the interfaces and changes in electronic structure due to the low thickness.

\section{CONCLUSIONS}

High quality bismuth iron garnet films were successfully grown on highly mismatched substrates. The discerning choice of the sample preparation conditions allowed to discriminate between the influence of strain and low dimension on the physical properties of BIG, taking into account at the same time the eventual contribution of bismuth nonstoichiometry, the sample morphology and the relaxation mode.

As for metallic systems, the low dimensional effects are responsible for the decrease of the magnetic characteristics of the films, such as Faraday rotation angle, saturation magnetization, and Curie temperature. The strain and the relaxation mode influence rather the critical thickness below which the material starts to behave as a low dimensional system. This critical thickness value is smaller for the lower lattice misfit between a substrate and a film.

\section{ACKNOWLEDGMENTS}

The authors acknowledge financial support from Ile-deFrance region for magnetic measurements (via NOVATECS C'Nano IdF Project No. IF-08-1453/R) and the CNRS-CEA
French microscopy network «METSA» for the microscopy characterization. The authors acknowledge K. March for her contribution to the sample preparation by FIB. A part of the TEM measurements has been conducted in the "Laboratorio de Microscopias Avanzadas" (LMA) at Instituto de Nanociencia de Aragon (INA)-Universidad de Zaragoza. The authors acknowledge the LMA-INA for offering access to their instruments and expertise. This work has been supported by the French National Research Agency under the "Investissement d'Avenir" programs, References Nos. ANR-10-EQPX-38-01 and ANR-10-EQPX-50 (TEMPOSNANOTEM). The authors acknowledge the "Conseil Regional Midi-Pyrénées" and the European FEDER for financial support within the CPER program. Finally, part of the DRX measurements were done at the CTU IEFMINERVE facility and partly supported by the RENATECH network and the General Council of Essonne.

${ }^{1}$ P. Zubko, S. Gariglio, M. Gabay, P. Ghosez, and J.-M. Triscone, Annu. Rev. Condens. Matter Phys. 2, 141 (2011).

${ }^{2}$ J. Cao and J. Wu, Mater. Sci. Eng., R 71, 35 (2011).

${ }^{3}$ D. G. Schlom, L.-Q. Chen, C. J. Fennie, V. Gopalan, D. A. Muller, X. Pan, R. Ramesh, and R. Uecker, Mater. Res. Bull. 39, 118 (2014).

${ }^{4}$ C. J. Fennie and K. M. Rabe, Phys. Rev. Lett. 97, 267602 (2006).

${ }^{5}$ J. Dvorak, Y. U. Idzerda, S. B. Ogale, S. Shinde, T. Wu, T. Venkatesan, R. Godfrey, and R. Ramesh, J. Appl. Phys. 97, 10C102 (2005).

${ }^{6}$ D. Fuchs, E. Arac, C. Pinta, S. Schuppler, R. Schneider, and H. v. Löhneysen, Phys. Rev. B 77, 014434 (2008).

${ }^{7}$ I. C. Infante, S. Lisenkov, B. Dupé, M. Bibes, S. Fusil, E. Jacquet, G. Geneste, S. Petit, A. Courtial, J. Juraszek, L. Bellaiche, A. Barthélémy, and B. Dkhil, Phys. Rev. Lett. 105, 057601 (2010).

${ }^{8}$ F. Sandiumenge, J. Santiso, L. Balcells, Z. Konstantinovic, J. Roqueta, A. Pomar, J. P. Espinós, and B. Martínez, Phys. Rev. Lett. 110, 107206 (2013).

${ }^{9}$ M. Kubota, K. Shibuya, Y. Tokunaga, F. Kagawa, A. Tsukazaki, Y. Tokura, and M. Kawasaki, J. Magn. Magn. Mater. 339, 63 (2013).

${ }^{10}$ J.-Y. Hwang, R. Morandotti, and A. Pignolet, Appl. Phys. Lett. 99, 051916 (2011).

${ }^{11}$ C. A. F. Vaz, J. A. C. Bland, and G. Lauhoff, Rep. Prog. Phys. 71, 056501 (2008).

${ }^{12}$ H. Yamahara, M. Mikami, M. Seki, and H. Tabata, J. Magn. Magn. Mater. 323, 3143 (2011).

${ }^{13}$ M. Kubota, A. Tsukazaki, F. Kagawa, K. Shibuya, Y. Tokunaga, M. Kawasaki, and Y. Tokura, Appl. Phys. Express 5, 103002 (2012).

${ }^{14}$ H. Wang, C. Du, P. C. Hammel, and F. Yang, Phys. Rev. B 89, 134404 (2014).

${ }^{15}$ L. Magdenko, E. Popova, M. Vanwolleghem, C. Pang, F. Fortuna, T. Maroutian, P. Beauvillain, N. Keller, and B. Dagens, Microelectron. Eng. 87, 2437 (2010)

${ }^{16}$ M. Inoue, R. Fujikawa, A. Baryshev, A. Khanikaev, P. B. Lim, H. Uchida, O. Aktsipetrov, A. Fedyanin, T. Murzina, and A. Granovsky, J. Phys. D: Appl. Phys. 39, R151 (2006).

${ }^{17}$ T. V. Murzina, I. E. Razdolski, O. A. Aktsipetrov, A. M. Grishin, and S. I. Khartsev, J. Magn. Magn. Mater. 321, 836 (2009).

${ }^{18}$ N. Adachi, K. Yogo, T. Ota, M. Takahashi, and K. Ishiyama, J. Appl. Phys. 109, 07A506 (2011).

${ }^{19}$ K. Satoh, T. Okuda, H. Yamamoto, H. Onodera, and I. Nakamichi, IEEE Transl. J. Magn. Jpn. 5, 1141 (1990).

${ }^{20}$ S. Kahl and A. M. Grishin, J. Magn. Magn. Mater. 278, 244 (2004).

${ }^{21}$ B. Vertruyen, R. Cloots, J. S. Abell, T. J. Jackson, R. C. da Silva, E. Popova, and N. Keller, Phys. Rev. B 78, 094429 (2008).

${ }^{22}$ E. Popova, N. Keller, M. Guyot, M.-C. Brianso, Y. Dumond, and M. Tessier, J. Appl. Phys. 90, 1422 (2001).

${ }^{23}$ E. Popova, N. Keller, F. Gendron, L. Thomas, M.-C. Brianso, M. Guyot, M. Tessier, and S. S. P. Parkin, J. Vac. Sci. Technol., A 19, 2567 (2001).

${ }^{24}$ Y. Dumont, N. Keller, E. Popova, D. S. Schmool, M. Tessier, S. Bhattacharya, B. Stahl, R. M. C. Da Silva, and M. Guyot, Phys. Rev. B 76, 104413 (2007). 
${ }^{25}$ W. Noun, E. Popova, F. Bardelli, Y. Dumont, R. Bertacco, A. Tagliaferri, M. Tessier, M. Guyot, B. Berini, and N. Keller, Phys. Rev. B 81, 054411 (2010).

${ }^{26}$ F. C. Frank and J. H. van der Merwe, Proc. R. Soc. London, Ser. A 198, 205 (1949); 198, 216 (1949); 200, 125 (1949).

${ }^{27}$ I. V. Markov, Crystal Growth for Beginners: Fundamentals of Nucleation, Crystal Growth and Epitaxy, 2nd ed. (World Scientific, Singapore, 2003).

${ }^{28}$ E. Popova, A. F. Franco Galeano, M. Deb, B. Warot-Fonrose, H. Kachkachi, F. Gendron, F. Ott, B. Berini, and N. Keller, J. Magn. Magn. Mater. 335, 139 (2013).

${ }^{29}$ E. Popova, L. Magdenko, H. Niedoba, M. Deb, B. Dagens, B. Berini, M. Vanwolleghem, C. Vilar, F. Gendron, A. Fouchet, J. Scola, Y. Dumont, M. Guyot, and N. Keller, J. Appl. Phys. 112, 093910 (2012).

${ }^{30}$ See https://www.physics.hu-berlin.de/en/sem/software for accessing the DigitalMicrograph plug-in to perform geometric phase analysis.

${ }^{31}$ Y. Zhu, C. Ophus, J. Ciston, and H. Wang, Acta Mater. 61, 5646 (2013).

${ }^{32}$ M. Deb, E. Popova, A. Fouchet, and N. Keller, J. Phys. D: Appl. Phys. 45, 455001 (2012).

${ }^{33}$ M. Deb, E. Popova, A. Fouchet, and N. Keller, Phys. Rev. B 87, 224408 (2013).

${ }^{34}$ P. Hansen and J.-P. Krumme, Thin Solid Films 114, 69 (1984).

${ }^{35}$ G. F. Dionne and G. A. Allen, J. Appl. Phys. 75, 6372 (1994).
${ }^{36}$ M.-Y. Chern, F.-Y. Lo, D.-R. Liu, K. Yang, and J.-S. Liaw, Jpn. J. Appl. Phys., Part 1 38, 6687 (1999).

${ }^{37}$ S. Geller, H. J. Williams, G. P. Espinosa, R. C. Sherwood, and M. A. Gilleo, Appl. Phys. Lett. 3, 21 (1963).

${ }^{38}$ P. Hansen, K. Witter, and W. Tolksdorf, Phys. Rev. B 27, 6608 (1983).

${ }^{39}$ E. Popova, B. Warot-Fonrose, F. Bonell, S. Andrieu, Y. Dumont, B. Berini, A. Fouchet, and N. Keller, Surf. Sci. 605, 1043 (2011).

${ }^{40}$ J. Narayan and B. C. Larson, J. Appl. Phys. 93, 278 (2003).

${ }^{41}$ M. J. Hÿtch, E. Snoeck, and R. Kilaas, Ultramicroscopy 74, 131 (1998).

${ }^{42}$ S. Leitenmeier, T. Körner, J. Griesbauer, M. Herbort, A. Heinrich, and B. Stritzker, J. Cryst. Growth 310, 5392 (2008)

${ }^{43}$ G. A. T. Allan, Phys. Rev. B 1, 352 (1970).

${ }^{44}$ E. Almahmoud, I. Kornev, and L. Bellaiche, Phys. Rev. B 81, 064105 (2010).

${ }^{45}$ Y. Li and K. Baberschke, Phys. Rev. Lett. 68, 1208 (1992).

${ }^{46} \mathrm{U}$. Köbler and A. Hoser, Renormalization Group Theory, Impact on Experimental Magnetism, Springer Series in Materials Science (SpringerVerlag, Berlin, Heidelberg, 2010).

${ }^{47}$ J. Cardy, Scaling and Renormalization in Statistical Physics (Cambridge University Press, 1996).

${ }^{48}$ J. T. Ou, F. Wang, and D. L. Lin, Phys. Rev. E 56, 2805 (1997).

${ }^{49}$ T. Ambrose and C. L. Chien, Phys. Rev. Lett. 76, 1743 (1996). 